\title{
A Genetic Linkage Map of the Hermaphrodite Teleost Fish Sparus aurata L.
}

\author{
Rafaella Franch, ${ }^{*}$ Bruno Louro, ${ }^{\dagger, 1}$ Matina Tsalavouta, ${ }^{\dagger, 1}$ Dimitris Chatziplis, ${ }^{\S}$ Costas S. \\ Tsigenopoulos, ${ }^{\ddagger}$ Elena Sarropoulou, ${ }^{\ddagger}$ Jenny Antonello, ${ }^{*}$ Andonis Magoulas, ${ }^{\ddagger}$ \\ Constantinos C. Mylonas, ${ }^{\ddagger}$ Massimiliano Babbucci, ${ }^{*}$ Tomaso Patarnello, ${ }^{*}, * *$ \\ Deborah M. Power, ${ }^{\dagger}$ Giorgos Kotoulas $^{\dagger}$ and \\ Luca Bargelloni*,2
}

*Department of Public Health, Comparative Pathology, and Veterinary Hygiene, University of Padova, 35020 Legnaro, Italy, ${ }^{\dagger}$ Centro de Ciencias de Mar, Universidade do Algarve, 8000-810 Faro, Portugal, ${ }^{\ddagger}$ Hellenic Center for Marine Research, 71003 Crete, Greece,

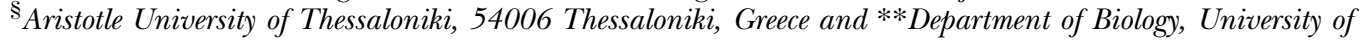
Padova, 35121 Padova, Italy

Manuscript received April 12, 2006

Accepted for publication August 7, 2006

\begin{abstract}
The gilthead sea bream (Sparus aurata L.) is a marine fish of great importance for fisheries and aquaculture. It has also a peculiar sex-determination system, being a protandrous hermaphrodite. Here we report the construction of a first-generation genetic linkage map for $S$. aurata, based on 204 microsatellite markers. Twenty-six linkage groups (LG) were found. The total map length was $1241.9 \mathrm{cM}$. The ratio between sex-specific map lengths was 1:1.2 (male:female). Comparison with a preliminary radiation hybrid (RH) map reveals a good concordance, as all markers located in a single LG are located in a single RH group, except for Ad-25 and CId-31. Comparison with the Tetraodon nigroviridis genome revealed a considerable number of evolutionary conserved regions (ECRs) between the two species. The mean size of ECRs was 182 bp (sequence identity 60-90\%). Forty-one ECRs have a known chromosomal location in the pufferfish genome. Despite the limited number of anchoring points, significant syntenic relationships were found. The linkage map presented here provides a robust comparative framework for QTL analysis in $S$. aurata and is a step toward the identification of genetic loci involved both in the determination of economically important traits and in the individual timing of sex reversal.
\end{abstract}

$\mathrm{T}$ HE gilthead sea bream (Sparus aurata L.) is a marine teleost fish that belongs to the family Sparidae. This family contains $>100$ species, which are divided into two large clades (groups A and B) according to the most recent molecular data (OrReLl and CARPENTER 2004). Sparids (porgies or sea breams) are demersal fish commonly found in temperate and tropical waters, with a maximum of diversity in the northeast Atlantic and the Mediterranean region (BAuchot and Hureau 1986), and they represent a key element of the coastal marine ecosystem. Sparids are also of great importance for fisheries and aquaculture, being excellent food fish, with high commercial value. S. aurata is the most prominent, with an average cultured production of 100 million metric tons per year. The great importance of the gilthead sea bream for marine aquaculture has fueled an increasing number of studies in many different areas such as immunology, endocrinology, bone morphology, and

Sequence data from this article have been deposited with the EMBL/ GenBank Data Libraries under accession nos. DQ851237-DQ851408.

${ }^{1}$ These authors contributed equally to this article.

${ }^{2}$ Corresponding author: Department of Public Health, Comparative Pathology, and Veterinary Hygiene, Viale dell'Università 16, Agripolis, University of Padova, I-35020 Legnaro, Italy.

E-mail: luca.bargelloni@unipd.it muscle physiology. Moreover, the genomic tool kit for $S$. aurata has been constantly improving, as a firstgeneration cDNA microarray was recently reported (SARROPOULOU et al. 2005), a preliminary radiation hybrid (RH) map has been constructed (SENGER et al. 2006), and a medium-scale expressed sequence tag (EST) sequencing project has been recently completed (A. CANARIO, personal communication). An improved RH map with $>1000$ markers (G. Kotoulas, personal communication) and an oligo array representing $>10,000$ unique transcripts (L. BARGELLONI, personal communication) are expected by the end of 2006. Therefore, the genome of the gilthead sea bream is rapidly becoming one of the best characterized among teleost species, apart from classical models such as Brachydanio rerio (zebrafish), Oryzias latipes (medaka), Takifugu rubripes (fugu), and Tetraodon nigroviridis (spotted green pufferfish). The gilthead sea bream belongs to the largest vertebrate order, the Perciformes, which comprises $>7000$ species including several fish families of great importance for fisheries and/or aquaculture (billfish, tuna, bass, cichlids, groupers, etc.) or for evolutionary studies, but none of the current fish model species (zebrafish, medaka, fugu, pufferfish). The development and implementation of genomic tools for the sea bream 
might therefore provide a link between these model species and the large perciform assemblage and give $S$. aurata the status of model marine organism.

The most striking feature of the family Sparidae is the variety of sex-determination mechanisms. There are gonochoristic species as well as hermaphrodite ones. The latter are sequential hermaphrodites and contains species that are either protandrous hermaphrodites (i.e., they mature first as males and then switch to females) or protogyneous hermaphrodites (i.e., they mature first as females and subsequently revert to the opposite sex). Sex reversal is made possible by the presence of a bisexual gonad, in which both testicular and ovarian tissues are present. During the male phase, the bisexual gonad has functional testicular and nonfunctional ovarian areas, whereas the inverse occurs in the female phase. Some other sparid species show a bisexual gonad, but never undergo sex reversal (incomplete hermaphrodites). Variation in sex determination is also present within the same species. In $S$. aurata, for instance, at 2 years of age, most individuals reverse to the female sex, through development and maturation of the ovarian portion of the gonad and regression of the testicular area. Not all male sea breams, however, seem to follow this pattern, because some animals either delay or never attain sex reversal, possibly in relation with social, environmental, and genetic factors (ZoHAR et al. 1978).

Here, the first linkage map for the gilthead sea bream is reported, on the basis of 204 microsatellite markers. So far, historical and technical reasons have meant that relatively little genetic improvement of aquacultured sea bream has occurred. Because of the peculiar features of several cultured marine species (mode of reproduction, generation time, origin of broodstocks) the use of molecular markers might be extremely helpful toward time- and cost-efficient breeding programs. Unfortunately, the application of molecular genetics for selection in fish aquaculture is still in its infancy. The linkage map presented in this work should provide a robust framework for implementation of QTL analysis and marker-assisted selection of important traits, especially those that are difficult to measure and/or have low heritability. A linkage map for $S$. aurata might also be used to identify genetic loci involved in a range of biological phenomena, such as adaptation to water temperature or salinity, stress and disease resistance, and the timing for sex reversal. Finally, the observed "comparability" of the gilthead sea bream map might facilitate genomic studies in other Sparidae as well as comparisons with well-characterized fish genomes.

\section{MATERIALS AND METHODS}

Mapping population: The mapping panel was produced by crossing a single sea bream male (contributing sperm) with a single female (contributing eggs). Both parents were part of the broodstock of the Hellenic Center for Marine Research (HCMR, Crete, Greece). As in other sea bream hatcheries, the
HCMR broodstock is mostly composed of animals directly caught from the wild. Therefore a negligible effect of captive breeding is expected on the genetic diversity of the broodstock. Even under farming conditions, reproduction in $S$. aurata occurs through mass spawning, where several females release their ova at the same time and several males contribute their sperm. To produce a mapping panel from a single pair of fish, a group of 3-year-old reproductively mature males $(n=5)$ and females $(n=4)$ was taken from a larger broodstock of HCMR in November, before the winter spawning season, and was placed in an outdoor $2-\mathrm{m}^{3}$ circular tank supplied with surface water of ambient salinity (38-40 psu) and temperature $\left(19^{\circ}-16^{\circ}\right)$. The tank was fitted with an overflow, automatic egg collector (ZoHAR et al. 1995), consisting of a cylindrical 500$\mu \mathrm{m}$ mesh placed cocentrically in a 200 -liter cylindrical tank. During the reproductive season, the egg collector was examined daily, to verify the onset of spawning and determine the time of spawning, which in gilthead sea bream is usually in the early afternoon, and it is at the same time throughout the season. A few days after the spawning season commenced, and once a regular daily spawning rhythm was established, all fish were removed from the tank at the expected time of spawning and were anesthetized in a bath of $0.4 \mathrm{ml} \mathrm{liter}^{-1}$ phenoxyethanol (Mylonas et al. 2005a). The females were examined for the presence of ovulated eggs, using gentle abdominal pressure. In the same way, two females found to have ovulated were stripped of their eggs, which were placed in clean, dry plastic bowls. Sperm from a different male was placed on top of the eggs of each female and mixed together using a plastic spoon, to obtain two different $F_{1}$, full-sib families. (One of the two families of the mapping panel showed half of the individuals with genotypes not compatible with the assumed parents. This was apparently due to the presence of contaminating sperm and/or eggs from a third individual. For this reason, only one family was used as a mapping panel and genotyped for all markers.)

At the same time, clean seawater from the incoming source was placed and the eggs were swirled gently for $2 \mathrm{~min}$ and were then allowed to stand for $5 \mathrm{~min}$. Then, the eggs were rinsed with plenty of clean seawater and were placed in separate egg incubators (500-liter cylindro-conical). Fertilization was verified $4 \mathrm{hr}$ later, by examining the eggs under a stereoscope and observing the onset of cell division. Two days later, and just before hatching, the eggs were moved into 500-liter circular tanks for larval rearing, which was undertaken using the intensive rearing method (MORETTI et al. 1999), until they reached an average of $3 \mathrm{~g}$. At this stage 200 animals were killed using an excess of anesthetics and stored at $-20^{\circ}$. A random sample of 50 individuals was collected and for each animal total genomic DNA was extracted using either a modified salting-out protocol (Miller et al. 1988) or a DNAeasy tissue mini kit (QIAGEN, Valencia, CA) following the manufacturer's instructions.

Microsatellite markers: Five different genomic libraries were constructed. Three repeat-enriched libraries were prepared following the FIASCO protocol (ZANE et al. 2002). Briefly, genomic DNA was digested with MseI to prepare the first two libraries, while TaqI was used for the third one. The corresponding adaptors (Mse or TaqI adaptors) were ligated to restricted DNA fragments, and the ligated products were PCR amplified using primers designed on the adaptors. The amplified DNA was then hybridized with a biotinylated oligonucleotide probe. For two libraries (one from MseI-digested DNA, one from TaqI-digested DNA) the probe was (AC) ${ }_{17}$. For the third one a (GATA $)_{8}$ oligo was used. After low- and highstringency washes, the repeat-enriched DNA was eluted and amplified with adaptor primers. An aliquot of the amplified DNA was cloned into a plasmid vector (pGEM-T Easy), using the pGEM-T Easy Vector System II (Promega, Madison, WI). 
Cloned inserts were amplified directly from individual bacterial colonies with universal vector primers (M13rev, caggaaaca gctatgac; T7, taatacgactcactataggg). The following experimental conditions were used: a final volume of $10 \mu \mathrm{l}$ reaction mixture contained 0.25 units of Taq DNA Polymerase (Promega), $1 \times$ Thermophilic DNA Polymerase buffer, magnesium free, $2.5 \mathrm{~mm} \mathrm{MgCl}_{2}, 0.14 \mathrm{~mm}$ dNTPs, 2.5 pmol of primer, and $\sim 50 \mathrm{ng}$ of bacterial DNA. The thermal profile, carried out in a GeneAmp 9700 (Applied Biosystems, Foster City, CA), included a preincubation step $\left(5 \mathrm{~min}\right.$ at $\left.95^{\circ}\right) ; 30$ cycles consisting of $1 \mathrm{~min}$ at $95^{\circ}, 1 \mathrm{~min}$ at $56^{\circ}, 1 \mathrm{~min}$ at $72^{\circ}$; and a final step $(10 \mathrm{~min}$ at $72^{\circ}$ ). One to two microliters of amplified DNA were sent without further purification to the sequencing core of the University of Padova (BMR Servizio Sequenziamento, CRIBI, Università di Padova).

Two additional microsatellite-enriched genomic libraries were prepared as described in TsigENopoulos et al. (2003), following a procedure first reported by EDwARDs et al. (1996). In summary, total sea bream DNA was digested with RsaI, ligated to RSaI-linkers, and, after denaturation, hybridized with a 5'-biotinylated (AC) 12 oligonucleotide probe. Repeatenriched DNA was then PCR amplified with primers designed on $R S a \mathrm{I}$ linkers and cloned into a pGEM-T vector as described above. Each recombinant clone was picked up into a 96-well plate in $100 \mu \mathrm{l}$ ultrapure water and incubated at $95^{\circ}$ for $5 \mathrm{~min}$. A $5-\mu l$ volume of each clone was added to the following PCR in a $20-\mu$ l volume of PCR mix (20 mm Tris- $\mathrm{HCl}, \mathrm{pH} 8.4,50 \mathrm{~mm}$ $\mathrm{KCl}, 1.5 \mathrm{~mm} \mathrm{MgCl}_{2}, 0.2 \mathrm{~mm}$ dNTPs, 0.5 unit Taq DNA polymerase) (Invitrogen Life Technologies, San Diego). Five picomoles each of primers AGE 1 (5'-GGAAACAGCTAGACCAT GAT) and AGE 2 (5'-TTGTAAAACGACGGCCAGTG) and of an $(\mathrm{AC})_{12}$ oligonucleotide were added. The thermal profile consisted of 30 cycles of $45 \mathrm{sec}$ at $93^{\circ}, 45 \mathrm{sec}$ at $55^{\circ}$, and $60 \mathrm{sec}$ at $72^{\circ}$. Plasmid DNA from clones that yielded two or more bands was extracted using the NucleoSpin Plasmid kit (MachereyNagel GmBH, Düren, Germany). DNA inserts were sequenced from both ends using SP6 and T7 primers, with a SequiTherm EXCEL II DNA sequencing kit-LC (Epicentre Technologies, Madison, WI), and run on a Li-Corp (Lincoln, NE) 4200 automated DNA sequencer.

The genomic sequences generated from the libraries described above were used to design primers with the Primer3 software (Rozen and Skaletsky 2000). In total, 171 polymorphic loci were obtained from the above libraries (GenBank accession nos. DQ851237-DQ851408). Six microsatellite loci published by BATARgias et al. (1999), one by LAUney et al. (2003), and six by BRown et al. (2005) were also used. Twenty sea bream sequences containing a microsatellite repeat that were available in GenBank (AJ418632, AJ418650-AJ418652, AJ418655-AJ418656, AJ418660, AJ418665, AJ418667, AJ418670AJ418673, AJ418675, AY138985, AF258447, and AF478568) were used to define primers, found polymorphic, and genotyped on the panel. One was located in the 5 '-flanking region of the growth hormone (GH) gene (AY138985, locus G1), one in the 3 '-untranslated region of the myostatin mRNA (AF258447, locus $2 \mathrm{G}$ ), one in the myogenic factor 1 gene (AF478568, locus G4), and three in ESTs (DN048390, DN048399, and DN048405).

Genotyping: PCR amplifications of microsatellite loci were performed under the following conditions: a final volume of $15 \mu \mathrm{l}$ reaction mixture contained 0.8 units of Taq DNA Polymerase (Promega), $1 \times$ Thermophilic DNA Polymerase buffer (magnesium free), $1 \mathrm{~mm} \mathrm{MgCl}_{2}, 0.070 \mathrm{~mm}$ dNTPs, $2.5 \mathrm{pmol}$ of primer, and $\sim 5-10 \mathrm{ng}$ of genomic DNA. The thermal profile included a predenaturation step (3 $\mathrm{min}$ at $\left.94^{\circ}\right)$; 33 cycles consisting of $1 \mathrm{~min}$ at $94^{\circ}, 50 \mathrm{sec}$ at $54^{\circ}$ or $58^{\circ}, 1 \mathrm{~min}$ at $72^{\circ}$; and a final step $\left(5 \mathrm{~min}\right.$ at $72^{\circ}$ ). Forward primers were fluorescently labeled using 6-FAM, HEX, or TAMRA. Up to nine different loci were loaded on an ABI PRISM 3100 DNA sequencer
(Applied Biosystems) with size standard Rox 400 (Applied Biosystems). Locus polymorphism was first scored for both parents and four individuals from the mapping panel. Markers considered informative (i.e., at least one parent was heterozygous) were then typed for all 50 individuals of the outcross. Individual genotypes were recorded using Genotyper 3.7 (Applied Biosystems) and transferred to an electronic spreadsheet.

Genomic DNA of six additional sparid species (Dentex dentex, Diplodus puntazzo, D. sargus, Lithognathus mormyrus, Pagellus bogaraveo, and Pagrus pagrus) was obtained as described for sea bream samples. Six individuals for each species were analyzed. Fifteen primer pairs were randomly selected from those amplifying polymorphic loci in S. aurata. PCR conditions and analysis of amplification products were as detailed above.

Accession numbers for each microsatellite locus are listed in supplemental information at http://www.genetics.org/ supplemental/. Details on primer sequences and product size are available at the SeaBream genome database (http://www. bridgemap.tuc.gr).

Map construction: Noninheritance errors were checked with LocusMap (GARBE and DA 2003). Such errors consisted of a 1-base difference in allele size due to rounding error to the unit. All noninheritance errors were checked by reevaluating the original chromatograms and corrected. A check for nonMendelian inheritance was also done using the prepare option of CRI-MAP. The linkage analysis and the building of the map were performed using the CRI-MAP 3.0 software (GREEN $e$ al. 1990). Assignment of the markers to linkage groups was performed by pairwise analysis of markers. Markers showing LOD scores $>3.0$ were assigned to the same linkage group. When there were more than three markers in a linkage group the order with the highest likelihood was selected from all the possible orders (up to six loci using the Flips6 option of CRIMAP). Any additional marker was assigned in the most likely position on a linkage group by looking at all possible orders of up to six adjacent loci in a linkage group. The maps were built assuming an equal recombination rate between sexes; however, the order of the loci was also checked (using the Flips6 option of CRI-MAP), assuming different recombination rates between sexes. No difference in the loci orders of the linkage groups was observed under the different assumptions of the recombination rate. The linkage distances for sex-average and sex-specific maps were estimated assuming the Kosambi mapping function.

Similarity searches: The T. nigroviridis genome (ver. 7.0) was downloaded from http://www.genoscope.cns.fr/externe/ English/Projets/Projet_C/data/genomique/assembly/masked/. The T. rubripes genome (ver. 4.0) was downloaded from http:// www.jgi.doe.gov/fugu/index.html. Individual chromosome sequences for zebrafish (ver. Zv6) as well as "nonchromosomal" genomic sequences were obtained from http://www. sanger.ac.uk/Projects/D_rerio/. Local BLAST and FASTA searches were carried out under default settings using individual sequences of sea bream genomic clones as queries. Repeat regions were masked. Hits with $e<10^{-5}$ were considered significant only when the same sea bream sequence showed similarity against both Tetraodon and Fugu genomes. Hits with $e<10^{-10}$ were always considered significant.

\section{RESULTS}

Sea bream-specific microsatellite markers: A total of 1199 clones were successfully sequenced from five enriched libraries, and 593 clones contained a repeat region $>18$ bp and flanking regions long enough to 
TABLE 1

Cross-amplification of microsatellite markers in other sparids

\begin{tabular}{ccccccc}
\hline Locus & $L m$ & $D d$ & $P b$ & $D s$ & $P p$ & $D p$ \\
\hline AD-05 & $+(6)$ & $+(1)$ & $+(5)$ & $+(8)$ & $+(1)$ & $+(4)$ \\
AD-20 & - & - & - & - & - & - \\
AD-12 & - & - & - & - & - & - \\
BD-03 & $+(1)$ & $+(2)$ & - & $+(4)$ & $+(1)$ & $+(1)$ \\
AD-21 & $+(1)$ & $+(1)$ & $+(4)$ & $+(1)$ & $+(3)$ & $+(1)$ \\
AD-66 & $+(5)$ & $+(4)$ & $+(3)$ & - & $+(1)$ & $+(6)$ \\
AD-54 & - & $+(1)$ & $+(6)$ & $+(7)$ & $+(1)$ & $+(1)$ \\
AD-86 & $+(6)$ & - & $+(1)$ & $+(4)$ & $+(1)$ & $+(1)$ \\
BD-14 & - & $+(1)$ & - & $+(4)$ & $+(1)$ & $+(1)$ \\
BD-48 & - & - & - & - & - & $+(2)$ \\
BD-68 & $+(4)$ & $+(1)$ & $+(1)$ & - & - & $+(3)$ \\
BD-36 & $+(1)$ & $+(1)$ & $+(3)$ & $+(1)$ & $+(1)$ & $+(4)$ \\
BD-86 & $+(4)$ & - & - & $+(6)$ & $+(6)$ & - \\
BD-29 & - & $+(1)$ & $+(1)$ & $+(1)$ & $+(1)$ & $+(1)$ \\
BD-72 & $+(7)$ & - & $+(6)$ & $+(1)$ & $+(1)$ & $+(1)$ \\
\hline
\end{tabular}

Species abbreviations are as follows: $D d$, Dentex dentex; $D p$, Diplodus puntazzo; Ds, D. sargus; Lm, Lithognathus mormyrus; Pb, Pagello bogaraveo; $\mathrm{Pp}$, Pagrus pagrus; and $\mathrm{Sa}$, S. aurata. In parentheses is the number of alleles found in each species sample.

design primers. The cutoff at $18 \mathrm{bp}$ was chosen after a preliminary analysis on 50 candidate loci. This analysis showed that all analyzed microsatellite loci with less than nine dinucleotide repeats were monomorphic, while $70 \%$ of loci with longer repeats were polymorphic on the mapping panel. A total of 204 polymorphic markers were optimized for amplicon size and fluorescent dye to analyze up to 9 loci in the same run. A random sample of 48 markers were genotyped on a larger number of individuals to estimate heterozygosity. Average observed heterozygosity was 0.69 (range 0.02-1). The mean number of alleles was 11.2 (range 2-30). A random sample of 15 microsatellite loci was also tested on six other sparid species to assess whether sea bream markers could be amplified across species. The percentage of successful amplifications was remarkably high, as 13 loci provided a visible product in at least one species other than $S$. aurata (Table 1). For most species, several loci showed more than one allele, despite the limited sample size (six individuals per species, originating from a single population). Therefore, preliminary results indicate that a good proportion of markers developed for the gilthead sea bream could be used for genetic analysis of other sparid species.

S. aurata linkage map: Significant linkages were identified for 198 microsatellite loci. Only 6 markers remained unlinked. Twenty-six linkage groups (LG) were found (Figure 1), with the number of markers per group ranging from 2 (LG22) to 17 (LG3). Seven loci were assigned to different linkage groups, but their position on the map could not be reliably defined. The total sex-averaged length of the map is $1241.9 \mathrm{cM}$. The largest linkage groups were LG3 and LG17, respectively, with 127.4 and $129.5 \mathrm{cM}$. Two linkage groups showed only 4 (LG21) and 2 loci (LG22) with no recombination among them. A total of 154 markers were genetically distinct. The distance between genetically distinct, contiguous markers ranged from 0.4 to 64.6 cM, with an average of $9.7 \mathrm{cM}$. Forty-eight percent of intermarker intervals are $<5 \mathrm{cM}$ long, 23\% ranged between 5 and $10 \mathrm{cM}, 17 \%$ varied between 10 and $20 \mathrm{cM}$, and $12 \%$ were $>20 \mathrm{cM}$. Only a slight difference in length was observed between the male map (1170.5 cM, supplemental Figure S1 in supplemental information at http://www.genetics.org/supplemental/) and the female one (1451.5 cM, supplemental Figure S2 in supplemental information at http://www.genetics.org/ supplemental/), with a ratio of 1:1.24 (male:female). Differences in length between male and female maps were especially observed in some linkage groups (i.e., $3,6,7,9,12,20$, and 25). Such differences might be partially due to the fact that both parents shared one allele (e.g., Bd-71-F of LG3, Eld-39-T of LG6, and Dld14-H of LG9) and/or one of the parents was homozygous at specific adjacent loci (e.g., B6b, P82, 172EP, and Fd-92-H of LG12; P19, Eld-13-H, and Eld-14-T of LG14; Dt23, Dld-03-T, Bt-14-F, and Fd-78-H of LG20; and Hd77-F of LG25). However, it should be noted that no bias toward one sex was observed in the number of informative loci, and when only loci that are informative in both parents $(n=75)$ were selected the average recombination fraction was still higher for the female sex $(0.11)$ than for the male $(0.10)$.

Comparison between sea bream linkage and the RH map: A preliminary RH map for the gilthead sea bream has been recently reported (SENGER et al. 2006). It contains 440 markers, including 121 microsatellite loci that have been mapped onto the linkage map presented here. The current RH map shows $28 \mathrm{RH}$ groups (supplemental information in SENGER et al. 2006). A comparison of the two maps reveals a good concordance, as nearly all markers located in a single LG are found in a single RH group. The observed correspondences are reported in Table 2. Only two of the markers (Ad-75 in LG9, CId-31 in LG23) are positioned in a different $\mathrm{RH}$ group (both in RH25) compared to markers in the same LG (Table 2). Remarkably, for 13 linkage groups (LG1, LG4, LG5, LG10, LG13, LG16, LG17, LG18, LG20, LG21, LG22, LG25, and LG26) the same marker order is found also in the corresponding RH group, for 6 of the linkage groups (LG2, LG6, LG11, LG14, LG19, and LG23) a single marker is placed in a different position from that observed in the RH group, and for the remaining 7 linkage groups more than one locus has a position that is not congruent between the two maps.

Comparison between the sea bream linkage map and the $\boldsymbol{T}$. nigroviridis genome: Similarity searches of $S$. aurata sequences against the genome sequence of the pufferfish $T$. nigroviridis revealed a relatively high 
LinkageGroup1

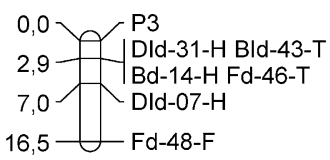

LinkageGroup2

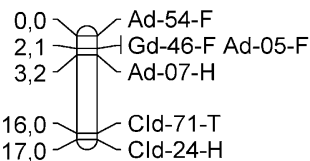

LinkageGroup3

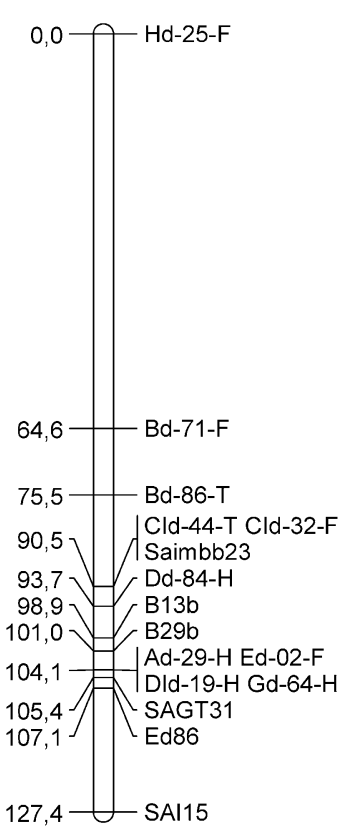

LinkageGroup4

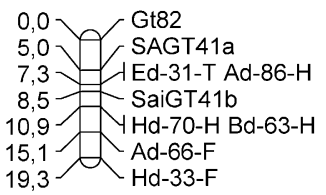

LinkageGroup5

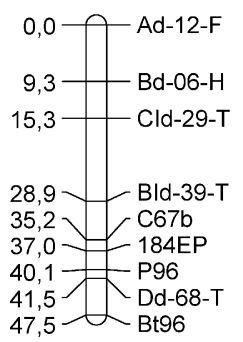

\section{LinkageGroup6 LinkageGroup7}

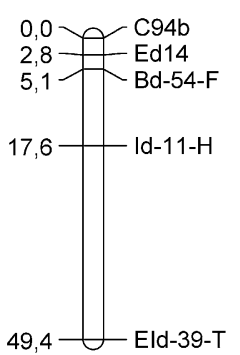

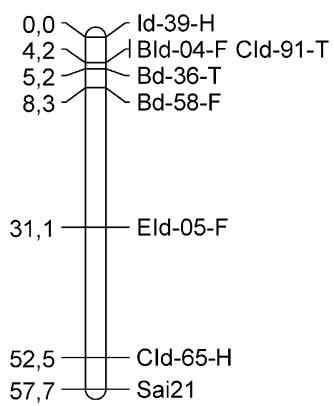

LinkageGroup8

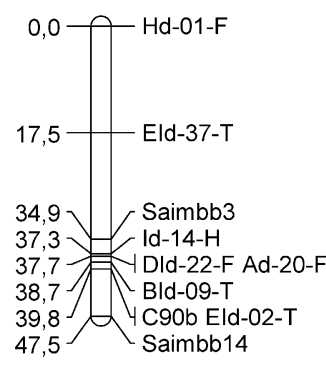

LinkageGroup9

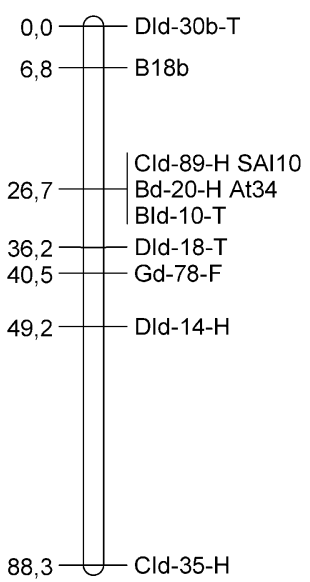

LinkageGroup10

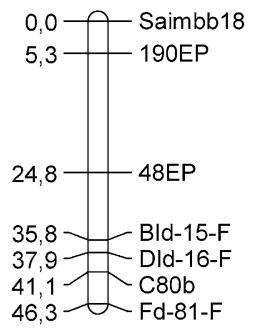

FiguRE 1.-Sex-averaged linkage map in Kosambi centimorgans of the gilthead sea bream consisting of 26 linkage groups.

percentage of evolutionary conserved regions (ECRs) between the two species. Thirty percent $(62 / 204)$ of sea bream microsatellite loci gave significant similarity to Tetraodon sequences, according to the criteria described in MATERIALS AND METHODS. The majority of Tetraodon-matching sequences $(58 / 62,93 \%)$ showed a significant match also against the fugu genome. The percentage of ECRs sharply decreases when sea bream genomic sequences are compared to the zebrafish genome $(18 / 204,9 \%)$. Quite remarkably, half of significant matches $(9 / 204)$ point to two different genomic regions of the zebrafish genome.

Using FASTA, the mean size of the aligned region was $182 \mathrm{bp}$ (range $68-410 \mathrm{bp}$ ), with sequence identity ranging from 60 to $>90 \%$. The majority of ECRs (41\%) are located in putatively transcribed regions [generally in the 3 '- and 5'-untranslated regions (UTRs) of known/predicted genes] of the Tetraodon genome, $27 \%$ are found in pufferfish introns, and $32 \%$ appear not to be associated with any known or predicted gene. Forty-one ECRs have a known chromosomal location in the Tetraodon genome. Despite the limited number of anchoring points, a comparison between the pufferfish genome and the sea bream linkage map reveals several syntenic regions (Table 3). For instance, five loci on LG18 (Gd60, CId-84, Saimbb20, Ad-21, and CId-52), spanning $3.1 \mathrm{cM}$, define a syntenic region on Tetraodon chromosome 13. Similarly, sea bream LG1 contains eight markers, of which three, DId-31, Fd-46, and Fd-48, with a total map length of $13.6 \mathrm{cM}$, have putative homologs 


\section{LinkageGroup11 LinkageGroup12}
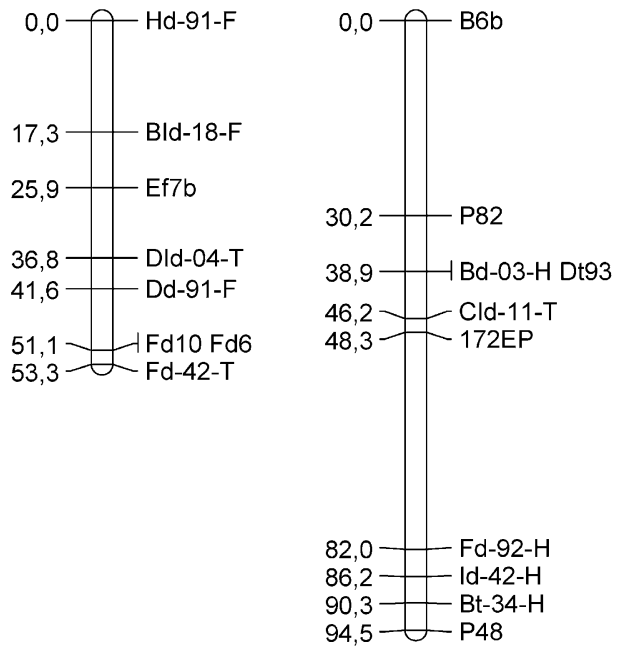

LinkageGroup13
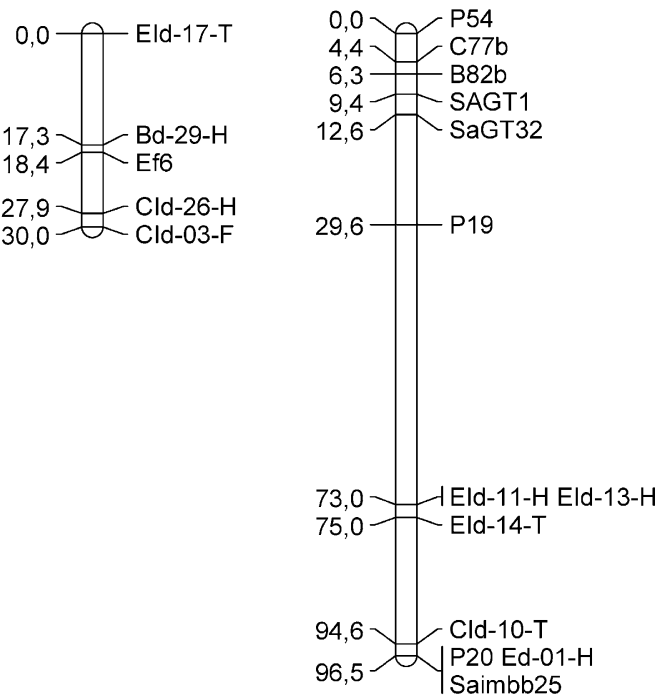

\section{LinkageGroup15}

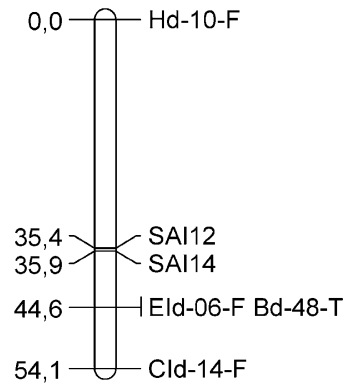

$54,1-$ Cld-14-F

\section{LinkageGroup16 LinkageGroup17}

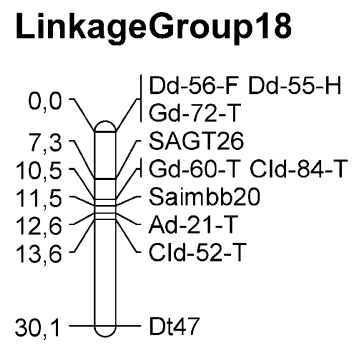

LinkageGroup19
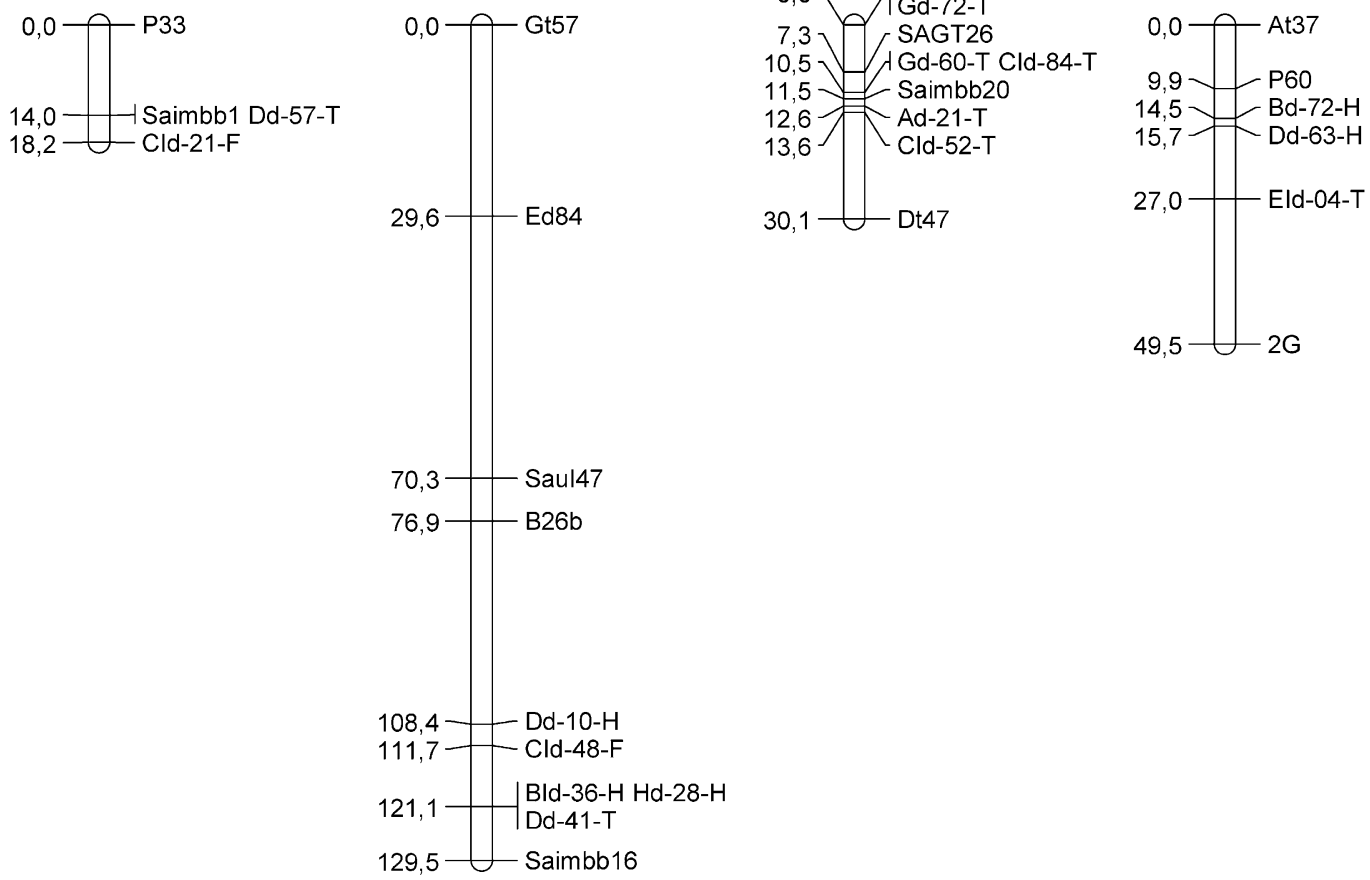

Figure 1.-Continued.

on chromosome 12 in the Tetraodon genome. Other, less extended correspondences are found (Table 3).

\section{DISCUSSION}

This is the first linkage map of the gilthead sea bream, a teleost fish that belongs to a large family of great importance for fisheries and aquaculture. The set of markers generated for the construction of the sea bream map is highly polymorphic and represents an important tool for further genetic studies in this spe- cies. Furthermore, preliminary evidence suggests that its utility could be extended to other species. As mentioned above, Sparidae are divided into two large clades (A and B in Orrell and Carpenter 2004). Crossspecies amplification of several microsatellite loci has been observed in this study as well as by BROwN et al. (2005) in sparid species belonging to both clades. The possibility of using the same markers across different species will speed up the construction of linkage maps in other sparids and allow easy comparisons between the different maps. 


\section{LinkageGroup20}

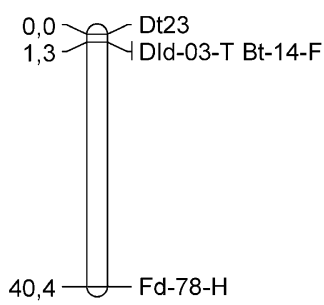

LinkageGroup21

$0,0-\bigcirc \mid \mathrm{Hd}-46-\mathrm{T}$ Eld-36-F Id-13-F DId-12-F
LinkageGroup22

$0,0-\bigcirc-1 \mathrm{Bd}-61-\mathrm{H}$ Dld-24-T
LinkageGroup23

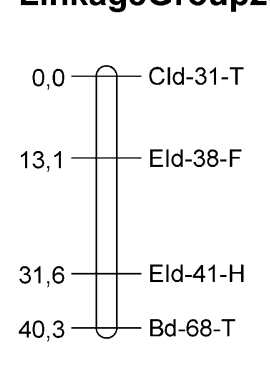

\begin{abstract}
.
\end{abstract}
LinkageGroup24

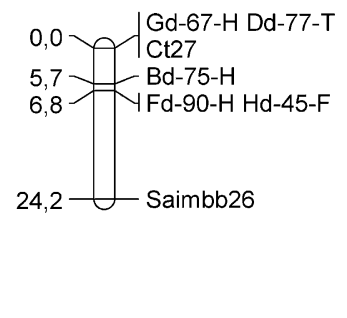

LinkageGroup25 LinkageGroup26

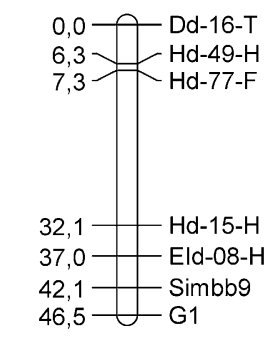

FigURE 1.-Continued.

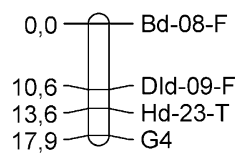

13,6- Hd-23-T 
TABLE 2

Oxford plot comparing the sea bream linkage and RH map

\begin{tabular}{|c|c|c|c|c|c|c|c|c|c|c|c|c|c|c|c|c|c|c|c|c|c|c|c|c|c|c|}
\hline \multirow[b]{2}{*}{ RH } & \multicolumn{26}{|c|}{ Linkage map } \\
\hline & 1 & 2 & 3 & 4 & 5 & 6 & 7 & 8 & 9 & 10 & 11 & 12 & 13 & 14 & 15 & 16 & 17 & 18 & 19 & 20 & 21 & 22 & 23 & 24 & 25 & 26 \\
\hline 1 & & & & & & & & & & & & & & & & & & & & 2 & & & & & & \\
\hline 2 & & & & & & & & & & & & & & & & & & & & & 3 & & & & & \\
\hline 3 & & & & & & & 8 & & & & & & & & & & & & & & & & & & & \\
\hline 4 & & & 10 & & & & & & & & & & & & & & & & & & & & & & & \\
\hline 5 & 4 & & & & & & & & & & & & & & & & & & & & & & & & & \\
\hline 6 & & & & & & & & & & 3 & & & & & & & & & & & & & & & & \\
\hline 7 & & & & & 5 & & & & & & & & & & & & & & & & & & & & & \\
\hline 8 & & & & & & & & & & & & & & & & & & & & & & & & 5 & & \\
\hline 9 & & 6 & & & & & & & & & & & & & & & & & & & & & & & & \\
\hline 10 & & & & & & & & 7 & & & & & & & & & & & & & & 2 & & & & \\
\hline 11 & & & & & & & & & & & & & & & & & & & & & & & & & 4 & \\
\hline 12 & & & & & & & & & & & 5 & & & & & & & & & & & & & & & \\
\hline 13 & & & & & & & & & & & & & & & & & & & 4 & & & & & & & \\
\hline 14 & & & & & & & & & & & & & & & & & 4 & & & & & & & & & \\
\hline 15 & & & & & & & & & & & & & & 4 & & & & & & & & & & & & \\
\hline 16 & & & & & & & & & & & & & & & 6 & & & & & & & & & & & \\
\hline 17 & & & & & & 3 & & & & & & & & & & & & & & & & & & & & \\
\hline 18 & & & & & & & & & & & & & 3 & & & & & & & & & & & & & \\
\hline 19 & & & & & & & & & & & & & & & & & & 7 & & & & & & & & 2 \\
\hline 20 & & & & & & & & & & & & & & & & 3 & & & & & & & & & & \\
\hline 21 & & & & 4 & & & & & & & & & & & & & & & & & & & & & & \\
\hline 22 & & & & & & & & & & & & 4 & & & & & & & & & & & & & & \\
\hline 23 & & & & & & & & & 7 & & & & & & & & & & & & & & & & & \\
\hline 24 & & & & & & & & & & & & & & & & & & & & & & & 4 & & & \\
\hline 25 & & & & & & & & & 1 & & & & & & & & & & & & & & 1 & & & \\
\hline 26 & & & & & & & & & & & & & & & & & & & & & & & & & & \\
\hline 27 & & & & & & & & & & & & & & & & & & & & & & & & & & \\
\hline 28 & & & & & & & & & & & & & & & & & & & & & & & & & & \\
\hline
\end{tabular}

sex-determining and sex-differentiating genes on meiotic recombination.

The 198 loci on the sea bream map are comprised in 26 linkage groups. The comparison with a preliminary sea bream RH map provides independent support for the observed LGs, as complete concordance is observed between RH groups and LGs, often with respect also to marker order within groups. Karyotype analysis of $S$. aurata indicates a haploid set of 24 chromosomes (CAtaudella et al. 1980; VitTuri et al. 1992). It is expected that, with the addition of more markers, some linkage groups will merge into larger ones, and the number of LGs should eventually match the number of chromosomes. Indeed, RH group 10 contains markers that are included either in LG8 or in LG22, so it is likely that these LGs will merge in a single group. Similarly, RH group 19 comprises loci that are located either on LG18 or on LG26 and these are also expected to give rise to one LG. The distance between loci Id-14 and Bd-61, which are contiguous on RH10, yet located, respectively, on LG8 and LG22 (Figure 1), is $80 \mathrm{cR}_{3000}$. One $\mathrm{cR}_{3000}$ on the sea bream RH map corresponds approximately to $140 \mathrm{~kb}$. Therefore, the physical distance between Id-14 and $\mathrm{Bd}-61$ is $\sim 11.2 \mathrm{Mb}$, yet these loci could not be linked on the genetic map. Even more striking, the RH map distance between CId-52 (LG18) and Hd-23 (LG22) is only $14 \mathrm{cR}_{3000}$, corresponding to $<2 \mathrm{Mb}$. It is well known that physical and genetic distances between syntenic loci could be quite different, because recombination events are not uniformly distributed along the same chromosome. It is possible that the region between loci on LG18 and LG22 is a preferential site for the formation of chiasmata, greatly inflating recombination frequency. This might significantly reduce the possibility of linking loci that are physically located on the same chromosome. For instance, in tilapia, the number of LGs (24) remained larger than the number of chromosomes (22), although this represents one of the most extensive linkage maps available for fishes and contains $>500$ microsatellite markers (LeE et al. 2005). The same might be true for the sea bream linkage map, because some of the unlinked markers from the current study may be from as yet unidentified linkage groups, which would become apparent with the addition of extra markers.

From all of the above it is clear that integration of different genomic data might be extremely useful for 
TABLE 3

Oxford plot comparing the sea bream linkage map and the Tetraodon genome

\begin{tabular}{|c|c|c|c|c|c|c|c|c|c|c|c|c|c|c|c|c|c|c|c|c|c|c|c|c|c|}
\hline \multirow[b]{2}{*}{ Tetraodon chromosome } & \multicolumn{25}{|c|}{ Linkage map } \\
\hline & 1 & 2 & 3 & 45 & 56 & 7 & 8 & 9 & 10 & 11 & 12 & 13 & 14 & 15 & 16 & 17 & 18 & 19 & 20 & 21 & 22 & 23 & 24 & 25 & 26 \\
\hline 1 & & & & & & & 3 & 2 & & & & & & & & & & & & & & & & & \\
\hline 2 & & 1 & & & & & & & & & & & & & & & & 2 & & & & & & & 1 \\
\hline 3 & & & & & & & & & & & & & & & & & & & & & & & & 1 & \\
\hline 4 & & & & & & & & & & & & & & & & & & & & & & & & & \\
\hline 5 & & & & & & & & & & & & & 1 & & & & & & & 2 & & & & & \\
\hline 6 & & & & & & & & & & & & & & & & & & & & & & & 2 & & \\
\hline 7 & & & & & & & & & & & & & & & & 1 & & & & & & & & & \\
\hline 8 & & & & & & & & & & & & & & & & 1 & & & & & & & & & \\
\hline 9 & 1 & & & & & & & & & & & & & & 1 & & & & & & & & & & \\
\hline 10 & & & & & & & & & & & & & & & & & & & & & & & & & \\
\hline 11 & & & & & & & & & & & & & & & & & & & & & & & & & \\
\hline 12 & 3 & & & & & & & & & & & & & & & & & & & & & & & & \\
\hline 13 & & & & & & & & & & & & & & & & & 5 & & & & & & & & \\
\hline 14 & & & & & & & & & & 1 & & & & & & & & & & & & & & & \\
\hline 15 & & & & & & & & & & & & & & & & 3 & & & & & & & & & \\
\hline 16 & & & & & & & & & & & & & & & & & & & & & & & & & \\
\hline 17 & & & & & & & & & 1 & & & & & & & & & & & & & & & & \\
\hline 18 & & & 2 & & & & & & & & & & & & & & & & & & & & & & \\
\hline 19 & & & & & & & & & & & 1 & & & & & & & & & & & & & & \\
\hline 20 & & & & 1 & & & & & & & & & & & & & & & & & & & & & \\
\hline 21 & & & & & & 1 & & & & & 1 & & & & & & & & & & & & & & \\
\hline
\end{tabular}

Two loci (Saimbb13 and Saimbb22) that remained unlinked showed a significant homology, respectively, with Tetraodon chromosome 7 and chromosome 2 .

the construction of robust genomic maps. To this end, comparative genomics offers a great opportunity, by means of transferring information from partially or completely sequenced genomes toward less characterized species. This approach is possible using anchoring sites, i.e., loci that are homologous between the species under comparison. In general, such loci are type I (coding) markers. Microsatellite (type II) markers generally reside in noncoding regions; therefore, it is generally thought that these loci are species specific. Apparently, this is not a general rule in teleost fish. In Cottus gobio, $77 / 171(45 \%)$ of microsatellite loci could be matched with Tetraodon genomic sequences (STEMSHORN et al. $2005)$. In the present study, $62 / 204(30 \%)$ of loci contain conserved sequence regions compared to the pufferfish genome. In tilapia, using a more stringent threshold for BLASTn searches $\left(e>10^{-8}\right), 61 / 545(11 \%)$ loci matched Tetraodon sequences (LEE et al. 2005). Using the same threshold 57/204 (28\%) sea bream sequences showed a significant match. The high sequence similarity in noncoding sequences observed might be only partially explained by the fact that these four species (T. nigroviridis, Tetraodontiformes; C. gobio, Scorpaeniformes; $O$. niloticus and $S$. aurata, Perciformes) are part of a highly derived teleost group, the Acantomorpha, which is thought to have originated quite recently (80100 million MYA, CHen et al. 2004). Indeed, when the distribution of sequence similarity for aligned genomic blocks was inferred from comparison between mouse and human genomes, which diverged 65-100 MYA, the frequency of "neutrally" conserved regions with average size of 200 bp was less than $15 \%$.

The presence of numerous highly similar noncoding segments (ECRs) is one of the most intriguing discoveries of comparative genomics (ALONI and LANCET 2005). ECRs are located either in noncoding transcribed regions or in the genomic vicinity of genes (promoters, introns), but are also found at large distances (e.g., 500 $\mathrm{kb})$ from the closest known gene. Similarly, in the present study, the majority of ECRs $(72 \%)$ were found either in transcribed regions or in introns of the green spotted pufferfish genome, but a substantial proportion could not be associated with any known gene. This result, however, should be taken with caution as the Tetraodon genome is only partially annotated. In a recent article (WoOLfe et al. 2005), nearly 1400 ECRs were identified, comparing the human and fugu genomes. The majority of these sequence fragments were experimentally demonstrated to have a functional role in the regulation of gene transcription. These ECRs, which have a mean size of $199 \mathrm{bp}$, also showed a peculiar nucleotide frequency pattern at their borders, with a strong bias toward A-T (WALTER et al. 2005). The average size of ECRs identified between pufferfish and sea bream is similar to that reported in other studies, whereas for C. gobio the mean size of conserved regions was shorter (40 bp, STEMSHORn et al. 2005). The observation of numerous ECRs associated with microsatellite loci might be 
explained as a consequence of generally high sequence conservation across the teleost fish genomes either due to strong functional constraints or because of a slow mutation rate. Alternatively, it might be possible that a biased sample of the genome was obtained during the construction of repeat-enriched genomic libraries. Both for S. aurata and for C. gobio, the same method was used for library preparation, which consists of digestion of genomic DNA with restriction enzymes, followed by adapter ligation (ZANE et al. 2002). Since some ECRs seem to have a high A-T frequency at their borders, the choice of restriction enzyme might increase the chance of isolating a conserved fragment; for example, the recognition site of MseI is TTAA whereas the one of TaqI is TCGA. In the present study, in the Msel library a higher percentage $(43.2 \%)$ of clones showed homology with a genomic sequence of Tetraodon compared to the TaqI library $(32.7 \%)$, although the difference was not significant in a Fisher's exact test $(P=0.2)$.

Irrespective of the reason for the high frequency of ECRs at microsatellite loci in teleost genomes and of their potential functional role, ECRs represent a unique opportunity to anchor completely sequenced fish genomes to genetic linkage maps of less characterized species. This, in turn, might be extremely useful toward a precise identification of genetic loci responsible for QTL or other phenotypes of interest.

We thank our colleagues at the Institute of Aquaculture at the Hellenic Center for Marine Research for producing the mapping panel and Nikos Papandroulakis for rearing juvenile fish and Jacques Lagnel for help with sequence data. This work was part of the European Union research project "Bridgemap, QLRT-2000-01797."

\section{LITERATURE CITED}

Aloni, R., and D. Lancet, 2005 Conservation anchors in the vertebrate genome. Genome Biol. 6: 115.

Batargias, C., E. Dermitzakis, A. Magoulas and E. Zouros, 1999 Characterization of six polymorphic microsatellite markers in the gilthead seabream, Sparus aurata (Linnaeus 1758). Mol. Ecol. 8: 897-898.

Bauchot, M.-L., and J.-C. Hureau, 1986 Sparidae, pp. 883-907 in Fishes of the North-eastern Atlantic and the Mediterranean, edited by P. J. P. Whitehead, M.-L. Bauchot, J.-C. Hureau, J. Nielsen and E. Tortonese. Unesco, Paris.

Brown, R. C., M. Tsalavouta, V. Terzoglou, A. Magoulas and B. J. MCANDREW, 2005 Additional microsatellites for Sparus aurata and cross-species amplification within the Sparidae family. Mol. Ecol. Notes 5: 605.

Cataudella, S., P. Perin Riz and L. Sola, 1980 A chromosome study of eight Mediterranean species of Sparidae (Pisces, Perciformes). Genetica 54: 155-159.

Chen, W. J., G. Orti and A. Meyer, 2004 Novel evolutionary relationship among four fish model systems. Trends Genet. 20: $424-431$.

Chistiakov, D. A., B. Hellemans, C. S. Haley, A. S. Law, C. S. TsigenOPOUlos et al., 2005 A microsatellite linkage map of the European sea bass Dicentrarchus labrax L. Genetics 170: $1821-1826$.

Coimbra, M. R. M., K. Kobayashi, S. Koretsugu, O. Hasegawa, E. Ohara et al., 2003 A genetic linkage map of the Japanese flounder, Paralichtus olivaceus. Aquaculture 220: 203-218.

Devlin, R. H., and Y. Nagahama, 2002 Sex determination and sex differentiation in fish: an overview of genetic, physiological, and environmental influences. Aquaculture 208: 191-364.
Edwards, K. J., J. H. A. Barker, A. Daly, C. Jones and A. Karp, 1996 Microsatellite libraries enriched for several microsatellite sequences in plants. Biotechniques 20: 758-760.

Garbe, J., and Y. DA, 2003 Locusmap User Manual Version 1.1. Department of Animal Science, University of Minnesota, Minneapolis/ St. Paul.

Gilbey, J., E. Verspoor, A. McLay and D. Houlihan, 2004 A microsatellite linkage map for Atlantic salmon (Salmo salar). Anim. Genet. 35: 98-105.

Green, P., K. Falls and S. Crooks, 1990 Documentation for CRIMAP version 2.4. Washington University School of Medicine, St. Louis.

Launey, S., F. Krieg, P. Haffray, J.-S. Bruant, A. Vannier et al., 2003 Twelve new microsatellite markers for gilted seabream (Sparus aurata L.): characterization, polymorphism and linkage. Mol. Ecol. Notes 3: 457-459.

Lee, B. Y., W. J. Lee, J. T. Streelman, K. L. Carleton, A. E. Howf et al., 2005 A second-generation genetic linkage map of tilapia (Oreochromis spp.). Genetics 170: 237-244.

Miller, S. A., D. D. Dykes and H. F. Polesky, 1988 A simple salting out procedure for extracting DNA from human nucleated cells. Nucleic Acids Res. 16: 1215.

Moen, T., B. Hoyneim, H. Munck and L. Gomez-Raya, 2004 A linkage map of Atlantic salmon (Salmo salar) reveals an uncommonly large difference in recombination rate between the sexes. Anim. Genet. 35: 81-92.

Moretti, A., M. Pedini Fernandez-Criado, G. Cittolin and R. Guidastri, 1999 Manual on Hatchery Production of Seabass and Gilthead Seabream. Food and Agriculture Organization of the United Nations, Rome.

Mylonas, C. C., G. Cardinaletti, I. Sigelaki and A. PolzonettiMAGNI, 2005a Comparative efficacy of clove oil and 2-phenoxyethanol as anesthetics in the aquaculture of European sea bass (Dicentrarchus labrax) and gilthead sea bream (Sparus aurata) at different temperatures. Aquaculture 246: 467-481.

Mylonas, C. C., L. Anezaki, P. Divanach, S. Zanuy, F. Piferrer et al., 2005b Influence of rearing temperature during the larval and nursery periods on growth and sex differentiation in two Mediterranean strains of Dicentrarchus labrax. J. Fish Biol. 67: $652-668$.

Orrell, T. M., and K. E. Carpenter, 2004 A phylogeny of the fish family Sparidae (porgies) inferred from mitochondrial sequence data. Mol. Phylogenet. Evol. 32: 425-434.

Rozen, S., and H. Skaletsky, 2000 Primer3 on the WWW for general users and for biologist programmers. Methods Mol. Biol. 132: 365-386.

Sakamoto, T., R. G. Danzmann, K. Gharbi, P. Howard, A. Ozaki et al., 2000 A microsatellite linkage map of rainbow trout $(\mathrm{On}$ corhynchus mykiss) characterized by large sex-specific differences in recombination rates. Genetics 155: 1331-1345.

Sarropoulou, E., G. Kotoulas, D. M. Power and R. Geisler, 2005 Gene expression profiling of gilthead sea bream during early development and detection of stress-related genes by the application of cDNA microarray technology. Physiol. Genomics 23: 182-191.

Senger, F., C. Priat, C. Hitte, E. Sarropoulou, R. Franch et al., 2006 The first radiation hybrid map of a perch-like fish: the gilthead seabream (Sparus aurata L). Genomics 87: 793-800.

Singer, A., H. Perlman, Y. L. Yan, C. Walker, G. Corley-Smith et al., 2002 Sex-specific recombination rates in zebrafish (Danio rerio). Genetics 160: 649-657.

Stemshorn, K. C., A. W. Nolte and D. Tautz, 2005 A genetic map of Cottus gobio (Pisces, Teleostei) based on microsatellites can be linked to the physical map of Tetraodon nigroviridis. J. Evol. Biol. 18: $1619-1624$.

Tsigenopoulos, C. S., B. Hellemans, D. A. Chistiakov, A. Libertini, G. Котоulas et al., 2003 Eleven new microsatellites of the sea bass (Dicentrarchus labrax L.). Mol. Ecol. Notes 3: 352354.

Uchida, D., M. Yamashita, T. Kitano and T. Iguchi, 2002 Oocyte apoptosis during the transition from ovary-like tissue to testes during sex differentiation of juvenile zebrafish. J. Exp. Biol. 205: 711-718.

Vitturi, R., A. Mazzola, E. Catalano and M. R. Lo-Conte, 1992 Karyotype characterization of four Mediterranean sparid 
fish (Pisces, Perciformes) using conventional and banding techniques. Cytobios 72: 107-115.

Waldbieser, G. C., B. G. Bosworth, D. J. Nonneman and W. R. Wolters, 2001 A microsatellite-based genetic linkage map for channel catfish, Ictalurus punctatus. Genetics 158: 727-734.

Walter, K., I. Abnizova, G. Elgar and W. R. Gilks, 2005 Striking nucleotide frequency pattern at the borders of highly conserved vertebrate non-coding sequences. Trends Genet. 21: 436-440.

Walter, R. B., J. D. Rains, J. E. Russell, T. M. Guerra, C. Daniels et al., 2004 A microsatellite genetic linkage map for Xiphophorus. Genetics 168: 363-372.

Woolfe, A., M. Goodson, D. K. Goode, P. Snell, G. K. McEwen et al., 2005 Highly conserved non-coding sequences are associated with vertebrate development. PLoS Biol. 3: e7.
Zane, L., L. Bargelloni and T. Patarnello, 2002 Strategies for microsatellite isolation: a review. Mol. Ecol. 11: 1-16.

Zohar, Y., M. Abraham and H. Gordin, 1978 The gonadal cycle of the captivity-reared hermaphroditic teleost Sparus aurata (L.) during the first two years of life. Ann. Biol. Anim. Biochem. Biophys. 18: 877-882.

Zohar, Y., M. Harel, S. Hassin and A. Tandler, 1995 Gilthead sea bream (Sparus aurata), pp. 94-117 in Broodstock Management and Egg and Larval Quality, edited by N. R. Bromage and R. J. RoBERTs. Blackwell Science, Oxford.

Communicating editor: C. HALEY 